\title{
A Maximum Power Point Tracking for a Photovoltaic System based on Optimum Sinusoidal Modulated Control Pulses
}

\author{
Mohammed Alqarni \\ Brunel University, UK \\ mohammed.alqarni@brunel.ac.uk
}

\author{
Mohamed K Darwish \\ Brunel University, UK \\ mohamed.darwish@brunel.ac.uk
}

\begin{abstract}
Photovoltaic systems have grown rapidly in the last few decades. This growth has enhanced research about this technology, focusing on reducing cost and improving efficiency. Maximum power point tracking (MPPT), which increases the overall efficiency of a PV system, is a main part of a PV system. In this paper, some MPPT methods have been critically reviewed. Fractional open-circuit voltage has been investigated. The approach of a separate PV array to obtain the open circuit voltage $\left(V_{o c}\right)$ is adopted. The proposed idea in this paper is based on optimising the control pulses for the DC-DC converter. The generation of control pulses is based on sinusoidal modulated pulse width modulation (SPWM). The output from the DC-DC converter is then fed into a low frequency square wave voltagefed inverter circuit. The output is a fine sinusoidal waveform, although the inverter operates at a low frequency. The block diagrams and the results are presented.
\end{abstract}

Index Terms - MPPT, PV system, Open circuit.

\section{INTRODUCTION}

Photovoltaic systems have grown rapidly in the last few decades. In 2008, the installation of PVs around the world almost doubled; there has been a continuous growth of approximately $50 \%$ in the past five years [1]. This has enhanced research about this technology, focusing on reducing cost and improving efficiency. Maximum power point tracking (MPPT) is a main part of a PV system. Because a PV's P-V curve is non-linear, there will be one exact point at the knee of the curve where the PV operates at its maximum (see Figure 1). This point moves as the curve shifts because of changing weather conditions, and the MPPT aims to track this point as soon as it moves [2]. Cost, speed, complexity, efficiency, measurement requirements and other factors vary in the different MPPT methods [3]. Perturb and observe is the most common method for tracking the MPP because it is simple and easy to implement. This method perturbs a new operating voltage value and compares it with the previous value. If the new perturbation is high, then the tracking is in the right direction and the next perturbation will be higher. As soon as the tracking captures a negative value, it should reverse. The same operation is repeated regularly until the MPP is found. The speed of this method depends on the perturbation step. If it is a large value, the tracking time will be short; however, the operating point is less likely to be on the exact MPP. A small value will be more accurate; however, more time will be needed. Oscillation and poor tracking under rapidly changing weather conditions are the disadvantages of this simple method [3],[4]. The incremental conductance method overcomes the oscillation problem in the $\mathrm{P} \& \mathrm{O}$ and shows better tracking quality. This method is based on the fact that at the MPP, $\mathrm{dp} / \mathrm{dv}=0$, positive on its left and negative on its right [3]. It shares the same perturb principle to move on the P-V curve; however, finding the MPP depends on the following equations:

$$
\begin{aligned}
& - \text {, at the MPP } \\
& -, \text { left of the MPP } \\
& \text { - , right of the MPP }
\end{aligned}
$$

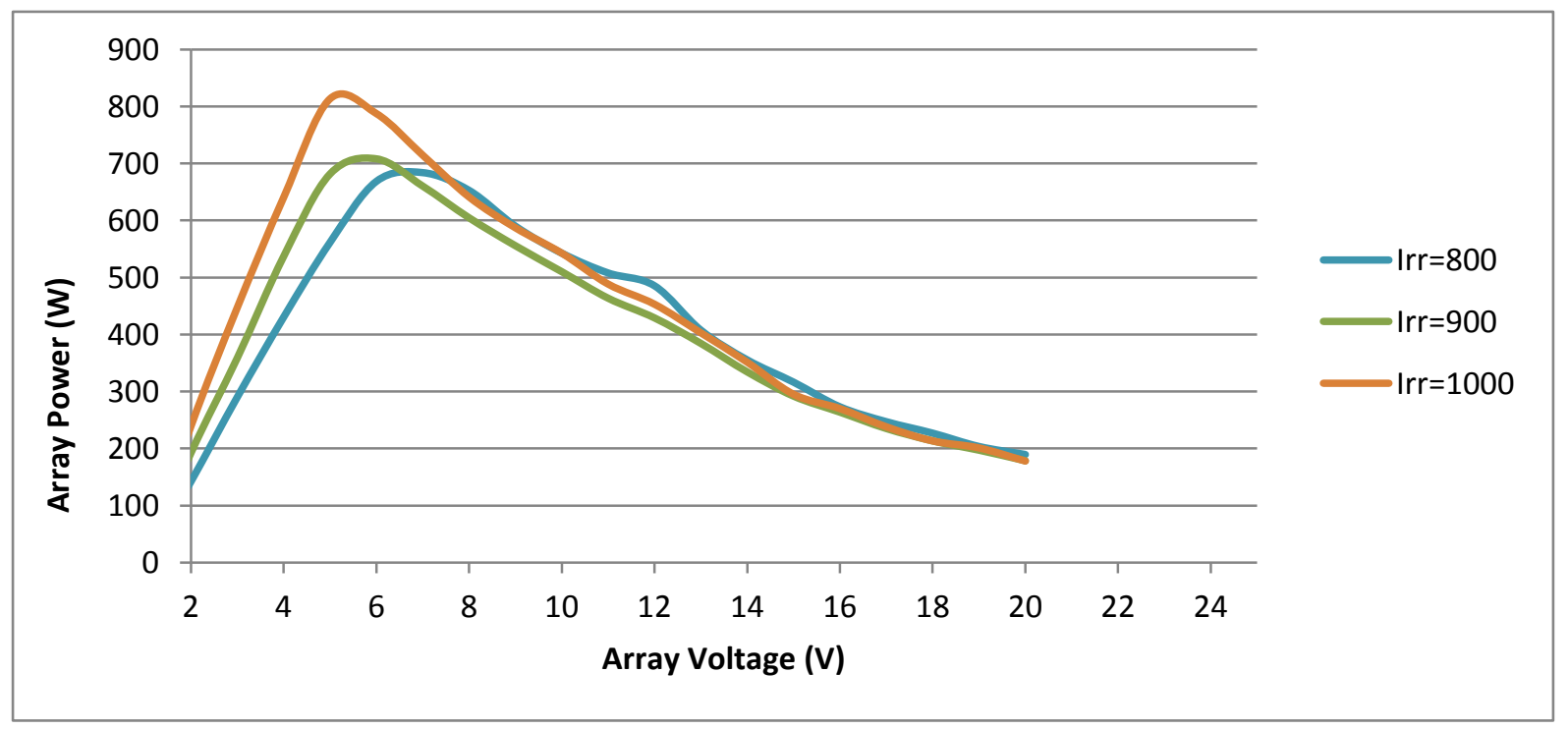

Fig.1. P-V characteristics of a PV module at different temperatures 


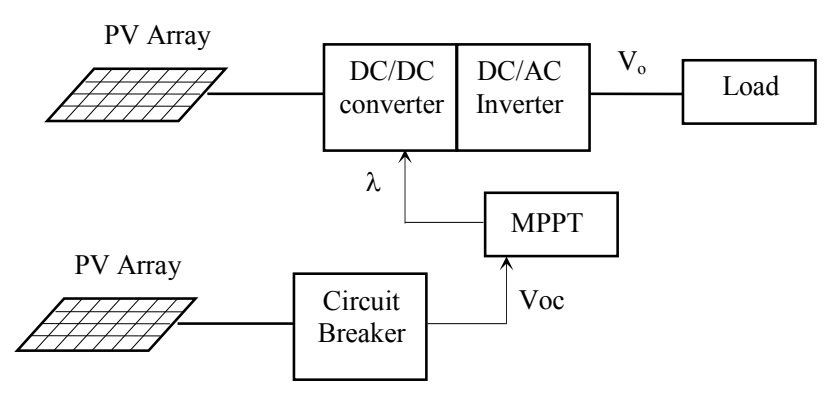

Fig.2. A block diagram of the proposed model

Other advanced methods are fuzzy logic and neural network, which can handle highly non-linear systems; however, these two methods require a more complex control algorithm [3],[4].

Fractional open-circuit voltage is a method that works because the open-circuit voltage of a PV array Voc and the voltage at maximum point Vmpp are near linear. Vmpp is a constant fractional of Voc. Literatures have allocated this fractional between 0.75 to 0.80 , which depends on the PV arrays properties and weather conditions [6]. The control unit measures Voc regularly by shutting down the power converter and adjusts the Vmpp depending on the following equation:

Vmpp $=\mathrm{K}$ Voc, where $0.75 \geq \mathrm{K} \leq 0.80$.

However, shutting down the power periodically results in power losses; this limitation could be overcome using another PV cell that faces the same condition (temperature and irradiance) and can be used to supply Voc as a reference and adjust the main PV voltage according to this value [3]. Usually, a PV does not operate at the exact MPP because the value of $\mathrm{K}$ is not accurate; however, this technique is cheap and easy to implement, which makes it one of the most commonly used MPPT techniques. The proposed idea in this paper, which benefited from the open-loop control method, was based on optimising control pulses for the DC-DC converter. The generation of control pulses was based on sinusoidal modulated pulse width modulation (SPWM). The output from the DC-DC converter was then fed into a low frequency square wave voltage-fed inverter circuit.

Figure 2 shows the block diagram for the proposed system. It consisted of two PV arrays, the main PV and the remote array, which fed the open-circuit data to the MPPT unit. Both arrays shared the same criteria and operated under identical weather conditions, ensuring that the tracking was accurate and the Vpv was the true Vmpp, or very close to it. The main $\mathrm{PV}$ array connected to the DC-DC received switching pulses from the MPPT algorithm. The converter was connected to the load through a DC-AC inverter.

\section{PROBLEM REVIEW}

The main disadvantage of the fractional open-circuit technique is power losses caused by the periodical shutting down of power to measure the Voc. However, the suggestion of using a separate array with the same properties overcame this problem. This suggestion was adopted in this paper for a higher efficiency tracking. The purposes of the DC-DC boost converter connected to the PV are to track the MPP and to step up the voltage. The output of this converter usually is a flat $\mathrm{DC}$ voltage, which is then connected to a high frequency DC-AC inverter. The inverter delivers the AC power to the grid or to the end-user. Having two high frequency conversion stages in the system increases the power losses caused by switching losses. However, if the inverter can operate at line frequency, this can improve a system's overall efficiency.

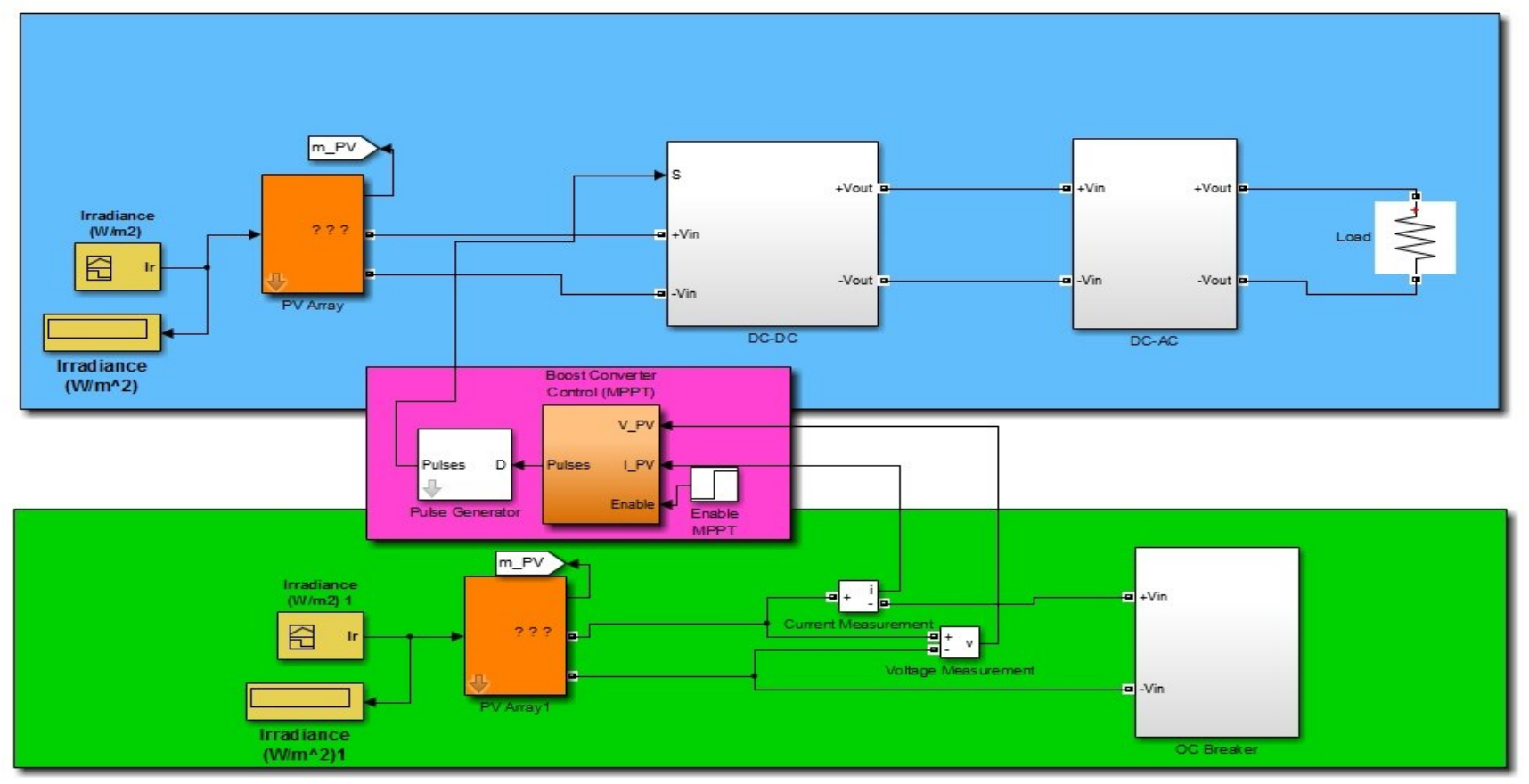

Fig.3. A block diagram of the proposed system 


\section{PROPOSED PV SYSTEM}

This paper has proposed an efficient system that connects a PV to the end-user, maintaining a high quality tracking process and low switching losses by controlling the switching pulses of the boost converter and running the $\mathrm{AC}$ inverter at line frequency. The fractional open-circuit algorithm was the MPPT method used in this system. To avoid power loss caused by switching off the circuit, a separate PV was built to measure the Voc. In this paper, instead of using a normal PWM to control the boost converter, a SPWM was used. The SPWM signal was built with specific adjustments to generate the control pulses that would reshape the PV output. This approach was supposed to transform the normal DC from the PV to a rectified sinusoidal waveform ready to be unfolded by the inverter. The inverter in the proposed system consisted of four switches and operated at line frequency to unfold the rectified sine wave and to inject a sinusoidal wave into the end-user. Figure 3 shows the proposed system on matlab/Simulink. The blue area was the main PV array connected to the DC-DC boost converter, which was connected to load through the DC-AC inverter. The green area was the remote PV cell that operated under the same irradiance and temperature conditions to obtain the Voc regularly. Between the two zones, the purple area contained the MPPT control, which took the Voc values and calculated the value of duty cycle, forcing the PV array to operate at Vmpp.

\section{THE SIMULATION}

Matlab/Simulink has a massive library, which has the capability to handle any power network. It has the ability to build any algorithm and design any power electronic component. Because of the aforementioned reasons, matlab/Simulink was the chosen software for this paper. As explained in the previous section, the proposed model contained two PV arrays, an MPPT, a DC-DC boost converter, an inverter and a load. At the beginning of this study, a DC voltage source was used to simplify the circuit. The DC-DC boost converter was designed properly to ensure it reshaped the DC and generated a rectified sine wave. Adjusting the value of the capacitor $(\mathrm{C})$ and the inductor (L) was critical in order to have the desired waveform. The converter operated at a $1000 \mathrm{~Hz}$ frequency. The switching pulses generated optimum sinusoidal modulated pulses that controlled the boost converter. The aim of this signal was to prepare the DC power to be converted to an $\mathrm{AC}$ in the next stage. In fact, this signal, besides stepping up the voltage also redrew the DC in a rectified sine wave. The next stage was the inverter, which consisted of four switches; all the switches operated at a $50 \mathrm{~Hz}$ line frequency. A $30 \mathrm{ohm}$ resistance load was connected to the other end of the inverter.

\section{THE SIMULATION RESULTS}

The proposed configuration was simulated in matlab/Simulink. Figure 4 shows the DC-DC boost converter and, as it was mentioned before, the values of $\mathrm{C}$ and $\mathrm{L}$ were

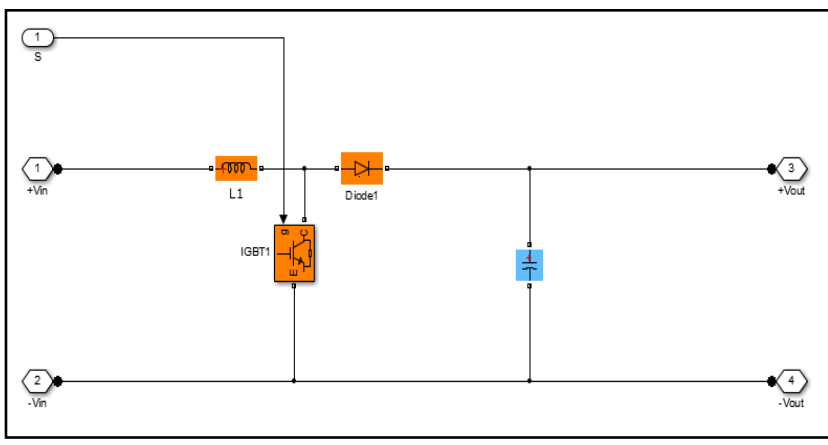

Fig.4. DC-DC boost converter

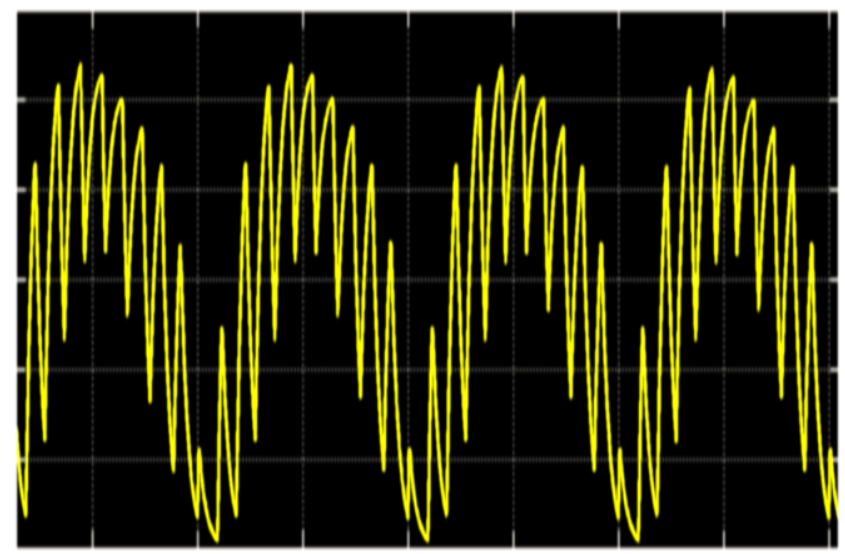

Fig.5. Boost converter output voltage

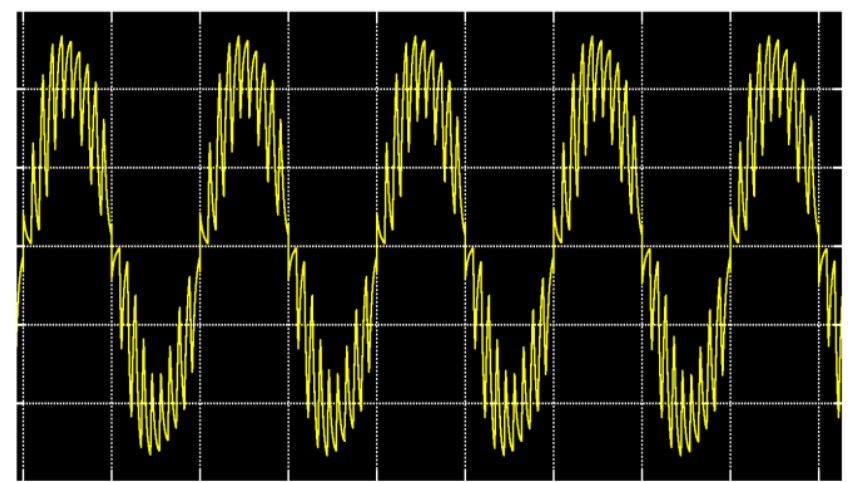

Fig.6. Load voltage 


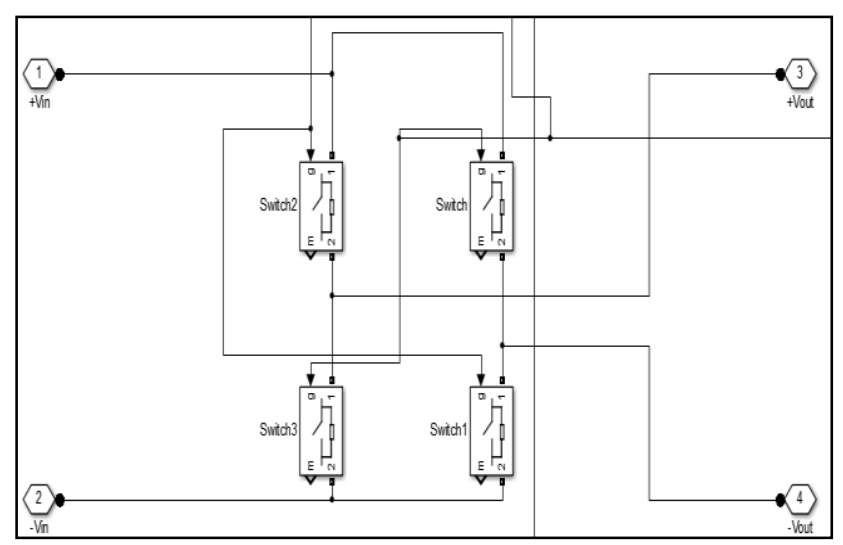

Fig.7. Inverter

optimised in order to get the desired output voltage shape. This output waveform is shown in figure 5 . The figure shows a rectified positive sine wave. This wave was shaped by control pulses, which were generated properly to obtain this output voltage form.

Figure 6 shows the final fine sinusoidal waveform, which was injected into the load. The inverter in this configuration operated on line frequency, and it flipped one cycle and left the other one in its order. Figure 7 shows the four switches that were used to make the inverter.

\section{CONCLUSIONS}

In this paper, short overview on MPPT techniques are presented. Then, a deeper investigation about the fractional open-circuit voltage method is discussed. This method is famous because it is easy to implement and does not require many sensors. However, switching the power on and off regularly results in power losses, which can be a disadvantage. The block diagram of the proposed system is presented. The system overcame power losses using a separate PV array under the same weather conditions. The aims of the proposed system were to track the MPP and to generate a rectified sine wave from the boost converter. The simulation results are shown and supported graphs are presented. Although a more in-depth study of the proposed technique should be performed, this study supports the validity of this technique.

\section{REFERENCES}

[1]. International Energy Agency [online]. $\begin{gathered}\text { Available: } \\ \text { http://www.iea.org/aboutus/faqs/renewableenergy/ }\end{gathered}$ 29/05/2014 at 11:00 pm.

[2]. M. Ngan and C. Tan, "A study of maximum power point tracking algorithms for stand-alone photovoltaic systems," Applied Power Electronics Colloquium, IEEE, PP.22,27, 2011.
[3]. T. Esram and P. L. Chapman, "Comparison of photovoltaic array maximum power point tracking techniques," Energy Conversion, IEEE Transactions, vol. 22, pp. 439,449, June 2007.

[4]. D. S'era, "Real-time modelling, diagnostics and optimised MPPT for residential PV systems," Ph.D. dissertation, Aalborg University, Denmark.2009.

[5]. S. Strache et al., "Maximum power point tracker for small number of solar cells connected in series," IECON 2012 - 38th Annual Conference on IEEE Industrial Electronics Society, NO. 978-1-4673-2420-5, PP. 5716- 5721, 25-28 Oct. 2012.

[6]. A. S. Khalifa And E. F. El-SaAdany, "Control of three phase GRID CONNECTED PHOTOVOLTAIC POWER SYSTEMS," HARMONICS AND QUALITY OF POWER (ICHQP), 2010 14TH INTERNATIONAL CONFERENCE, PP. 1, 7, 26-29 SEPT. 2010.

[7]. S. B. Kjær, "Design and control of an inverter for photovoltaic applications," Ph.D. dissertation, Aalborg University, Institute of Energy Technology, January 2005. 